\title{
De novo mass spectrometry peptide sequencing with a transformer model
}

\author{
Melih Yilmaz ${ }^{1}$, William E. Fondrie ${ }^{2}$, Wout Bittremieux ${ }^{3}$, Sewoong $\mathrm{Oh}^{1}$, and William \\ Stafford Noble ${ }^{4,1}$ \\ ${ }^{1}$ Paul G. Allen School of Computer Science and Engineering, University of Washington \\ ${ }^{2}$ Talus Bioscience \\ ${ }^{3}$ Skaggs School of Pharmacy and Pharmaceutical Science, University of California San Diego \\ ${ }^{4}$ Department of Genome Sciences, University of Washington
}

\begin{abstract}
Tandem mass spectrometry is the only high-throughput method for analyzing the protein content of complex biological samples and is thus the primary technology driving the growth of the field of proteomics. A key outstanding challenge in this field involves identifying the sequence of amino acids - the peptide - responsible for generating each observed spectrum, without making use of prior knowledge in the form of a peptide sequence database. Although various machine learning methods have been developed to address this de novo sequencing problem, challenges that arise when modeling tandem mass spectra have led to complex models that combine multiple neural networks and post-processing steps. We propose a simple yet powerful method for de novo peptide sequencing, Casanovo, that uses a transformer framework to map directly from a sequence of observed peaks (a mass spectrum) to a sequence of amino acids (a peptide). Our experiments show that Casanovo achieves state-of-the-art performance on a benchmark dataset using a standard cross-species evaluation framework which involves testing with out-of-distribution samples, i.e., spectra with never-before-seen peptide labels. Casanovo not only achieves superior performance but does so at a fraction of the model complexity and inference time required by other methods.
\end{abstract}

\section{Introduction}

Tandem mass spectrometry provides a high-throughput framework for identifying and quantifying proteins in complex biological samples, but determining the exact protein content from observed mass spectra at scale remains a challenge. At the core of this challenge is the spectrum identification problem, in which we are given an observed mass spectrum and the associated mass and charge of the peptide (known as the precursor) that is responsible for generating the spectrum, and we must infer the amino acid sequence of the precursor peptide. The standard method for solving this problem is enumerative, scoring each observed spectrum with respect to a list of candidate peptides (i.e., peptides whose masses are close to the observed precursor mass associated with the spectrum) and reporting the best-scoring peptide-spectrum match (PSM) per spectrum.

However, the drawback to any database search methodology is that it requires that we specify a priori which peptides might occur in the sample. Such an approach is often sensible when analyzing samples from a species, such as human, with a well-characterized genome sequence. However, relying on a database prevents the detection of unexpected peptide sequences, such as those that arise from genetic variation. A sequence database also cannot be used for the analysis of some types of immunopeptidomics data, for example, when a phenomenon known as $\mathrm{V}(\mathrm{D}) \mathrm{J}$ recombination results in peptides whose sequences are not encoded in the genome [1, in antibody sequencing [2, or in vaccine development when searching for bacterial peptides present on the surface of infected cells [3]. Finally, constructing an accurate database for metaproteomic analyses, such as the human microbiome or environmental samples, is nearly impossible [4. Such settings require de novo peptide sequencing from the acquired mass spectra. 


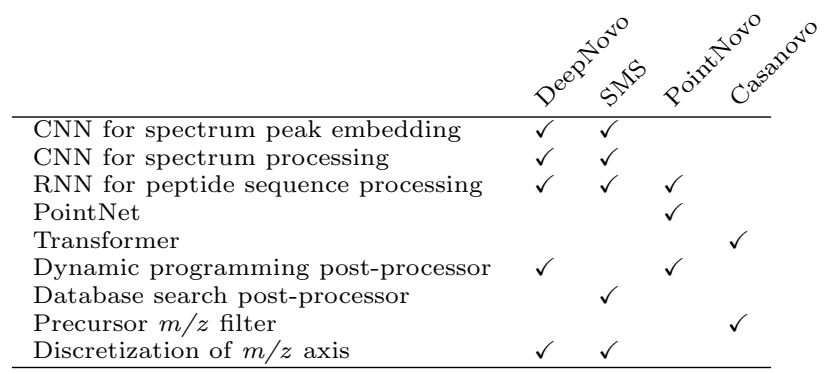

Table 1: Comparison of deep learning methods for de novo peptide sequencing. Casanovo introduces a simpler yet more powerful architecture of a transformer for de novo peptide sequencing.

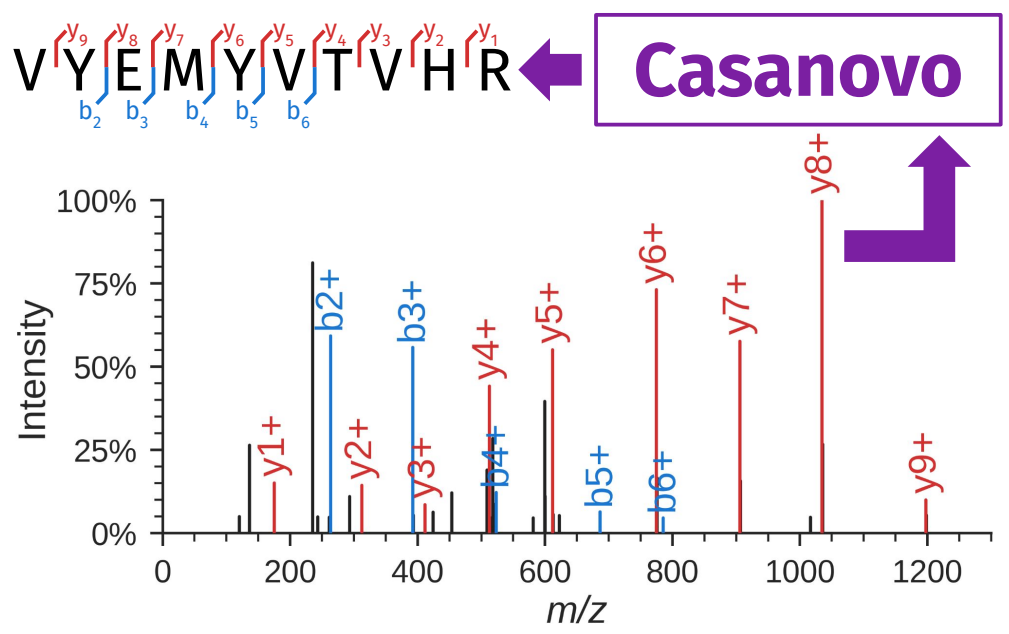

Figure 1: Casanovo performs de novo peptide sequencing. Casanovo takes as input an observed spectrum and produces the sequence of the generating peptide (e.g., VYEMYVTVHR). In the spectrum, peaks corresponding to b- and y-ions of the associated peptide are in color, and black peaks correspond to unexpected fragmentation events or noise. The spectrum annotation was created using spectrum_utils [13.

Early de novo methods used heuristic search [5] or dynamic programming [6] 8] to score peptide sequences against each observed spectrum. Machine learning has provided state-of-the-art performance on this task since 2015 [9, and recent methods employ deep neural networks 2, 10 12. Although de novo search tools are improving, there is still a long way to go. The most recent report [10] suggests that state-of-the-art methods achieve peptide-level recall (i.e., the percentage of spectra with the correctly assigned peptide) of 39-60\%, depending on the dataset. However, this percentage is calculated only with respect to ground truth spectra that, by definition, were previously identified with high confidence by a database search.

Additionally, all of these methods involve complicated modeling schemes (Table 1) featuring different neural networks for different sub-tasks such as convolutional neural networks (CNNs) for spectrum peak embedding and spectrum processing, and recurrent neural networks (RNNs) for peptide sequence processing. These methods also include complex post-processing steps that involve either matching the predicted peptide mass with the spectrum's observed precursor mass using dynamic programming or refining low confidence predictions with a database search-like procedure. The necessity of discretizing the mass-to-charge $(\mathrm{m} / z)$ axis of the mass spectra also remains a setback for all existing methods except PointNovo, necessitating a trade-off between low binning resolution (hence low sequencing accuracy) and higher model complexity (hence longer inference time).

In this work, we propose Casanovo, a transformer framework for de novo peptide sequencing (Figure 1). Casanovo uses the self-attention mechanism to translate directly from a variable-length sequence of observed spectrum peaks to a variable-length sequence of amino acids, analogous to a neural machine translation model in the natural language processing setting. Importantly, Casanovo takes individual spectrum peaks, 
together with the precursor mass and charge, as input, without resorting to discretization of the $m / z$ axis, and learns to predict the generating peptide sequence in a supervised setting in which ground truth sequences are obtained with database search. Unlike existing methods, Casanovo does not employ an additional RNN to process peptide sequences and replaces the dynamic programming post-processing step with a simple delta mass filter, offering a simpler yet more powerful framework.

We train and evaluate our model on a multi-species benchmark dataset using an established cross-validation framework which involves testing with out-of-distribution samples, i.e., spectra with never-before-seen peptide labels, through cross-species prediction. Our experiments show that Casanovo predicts peptide sequences with markedly higher precision and roughly comparable recall relative to the state-of-the-art methods, DeepNovo and PointNovo, and does so using a model with fewer parameters and requiring much shorter inference time. We also provide evidence that the nominally incorrect predictions made by Casanovo are enriched for spectra that are generated by a mixture of two different peptides, suggesting that Casanovo's reported error rate may be an overestimate. Finally, we benchmark several variants of the model to demonstrate the robustness of some of our modeling choices.

\section{Background: De novo peptide sequencing}

Because tandem mass spectrometry analysis, and particularly de novo peptide sequencing, is rarely discussed in the machine learning literature, we begin with a brief overview of the problem domain. Additional background is available in several review articles [14, 15.

A tandem mass spectrometer measures mass-to-charge $(\mathrm{m} / z)$ ratios of charged peptides in a two-scan process. The first scan (MS1) measures the $m / z$ of the intact peptide (also known as the precursor); the peptide is then fragmented and the resulting fragments are analyzed in a secondary scan (MS2). This MS2 scan is carried out on a population of (ideally) homogeneous peptide sequences, each of which is randomly fragmented at one location along the peptide backbone. As a result, the fragmentation scan contains peaks that correspond to prefixes (called b-ions) and suffixes ( $y$-ions) of the peptide, each with an associated charge state. Thus, the primary data object, the MS2 spectrum, consists of a bag of peaks, where each peak is characterized by an $m / z$ value and its associated intensity (Figure 11). The intensity is unitless but is monotonically related to the number of ions that generated the observed peak. The $m / z$ value is measured with extremely high precision, often better than 10 parts-per-million (ppm), whereas the intensity is measured much less precisely. In practice, each spectrum contains around 100 peaks, with considerable variation (10-476 in the data analyzed here). A mass spectrometer produces MS2 spectra at a rate of $\sim 20-40 \mathrm{~Hz}$, and a typical mass spectrometry run lasts 30-60 minutes, yielding on the order of 36,000-144,000 spectra in a single run.

The first analysis task that this data presents is the spectrum identification problem, in which each observed MS2 spectrum must be linked with the sequence of the peptide responsible for generating it. The primary constraint is that the peptide mass must lie within a specified tolerance of the observed precursor mass associated with the spectrum. This task is challenging because some of the expected b-ion and y-ion peaks may be missing, and some additional peaks may appear in the spectrum, created by losses of small molecular groups during fragmentation or by multiple cleavage events occurring on the same peptide. Spectra also contain noise, including experimental noise from the instrument as well as chemical noise produced by contaminants, other peptides, or non-peptide molecules.

In practice, spectrum identification is most commonly solved using database search, in which candidate peptides are selected from a given database of peptides [16. The database typically contains all of the peptide sequences encoded in the genome of the species from which the sample is derived. De novo peptide sequencing, on the other hand, considers all possible peptide sequences and is useful for identifying sequences that arise from genetic variation, recombination in some immune system settings, vaccine design, antibody sequencing, or in the analysis of metaproteomic samples from many different types of microorganisms.

\section{Related Work}

Early de novo methods used heuristic search (Lutefisk [5]) or dynamic programming (PEAKS [6] and SHERENGA [7]) to score peptide sequences against each observed spectrum. The PepNovo algorithm [8] uses a similar dynamic programming approach but employs a probabilistic score function that takes into 
account various chemical and physical rules governing peptide fragmentation. This model is closely related to the hidden Markov model that is, to our knowledge, the first application of machine learning to the de novo peptide sequencing task [17. A decade later, the Novor algorithm [9] achieved improved performance by using a decision tree as the score function in a dynamic programming algorithm. The first deep neural network algorithm for de novo peptide sequencing, DeepNovo [2], combines two different network architectures - a convolutional neural network and a long short term memory (LSTM) network - each of which aims to predict the subsequent amino acid, given a spectrum and a peptide prefix. These two scores are combined in a dynamic programming procedure to yield the predicted peptide sequence. The recently described SMSNet algorithm [12] uses a network architecture similar to that of DeepNovo but also offers a post-processing step in which low-confidence amino acids are replaced by making use of a user-supplied peptide database. A competing method, pNovo 3 [11, works in three steps: (1) a traditional dynamic programming approach generates a set of candidate peptides for a given spectrum, (2) a previously described deep learning model, pDeep [18, predicts a theoretical spectrum for each candidate, and (3) a ranking support vector machine ranks the candidate peptides, based on features extracted by comparing the observed and theoretical spectra. Finally, PointNovo [10] is an improved version of DeepNovo which focuses specifically on handling high-resolution mass spectrometry data by using an order-invariant network architecture [19].

\section{Methods}

Transformers are highly capable of learning contextualized representations and modeling sequential data [20, with a variety of successful applications to biological sequences [21, 22. In this context, de novo peptide sequencing can be formulated as a sequence-to-sequence learning problem where variable-length sequences of observed spectra peaks are translated into variable-length sequences of amino acids. The main contribution of this paper is to propose a transformer-based de novo peptide sequencing framework, Casanovo, which provides a unified solution to de novo peptide sequencing sub-tasks such as learning latent representations for spectra, spectrum processing and peptide sequence processing, which existing methods tackle separately through more complex modeling schemes.

\subsection{Casanovo}

Casanovo consists of a transformer encoder and decoder stack as described in [20, which are respectively responsible for learning latent representations of the input spectrum peaks and decoding the amino acid sequence of the spectrum's generating peptide (Figure 22). The encoder takes $d$-dimensional spectrum peak embeddings as input and outputs $d$-dimensional latent representation vectors for each peak. Subsequently, the decoder takes as input these representations of prefix amino acids, coupled with a $d$-dimensional precursor embedding encapsulating precursor $\mathrm{m} / z$ and charge information, to predict the next amino acid in the peptide sequence. We discuss different aspects of our modeling strategy in detail below.

\subsubsection{Input embeddings}

Each spectrum $S=\left\{\left(m_{j}, I_{j}\right)\right\}_{j=1}^{N}$ is a bag of peaks, where each peak $\left(m_{j}, I_{j}\right)$ is a 2-tuple representing the $\mathrm{m} / z$ value and intensity of the peak. The $m / z$ value and intensity are embedded separately before being summed to yield the input peak embedding. We use a fixed, sinusoidal embedding [20] to project each $\mathrm{m} / z$ value to a $d$-dimensional vector, the $m / z$ embedding $f$. Specifically, we create the $m / z$ embedding from an equal number of sine and cosine waveforms spanning the wavelengths from 0.001 to $10,000 \mathrm{~m} / z$, where each feature in the embedding $f_{i}$ is

$$
f_{i}= \begin{cases}\sin \left(m_{j} /\left(\frac{\lambda_{\max }}{\lambda_{\min }}\left(\frac{\lambda_{\min }}{2 \pi}\right)^{2 i / d}\right)\right), & \text { for } i \leq d / 2 \\ \cos \left(m_{j} /\left(\frac{\lambda_{\max }}{\lambda_{\min }}\left(\frac{\lambda_{\min }}{2 \pi}\right)^{2 i / d}\right)\right), & \text { for } i>d / 2\end{cases}
$$

where $\lambda_{\max }=10,000$ and $\lambda_{\min }=0.001$. These input embeddings provide a granular representation of high-precision $m / z$ information and, similar to relative positions in the original transformer model [20], may help the model attend to $\mathrm{m} / z$ differences between peaks, which are critical for identification of amino acids in the peptide sequence. The intensity, which is measured with lower precision than the $m / z$ value, is embedded 


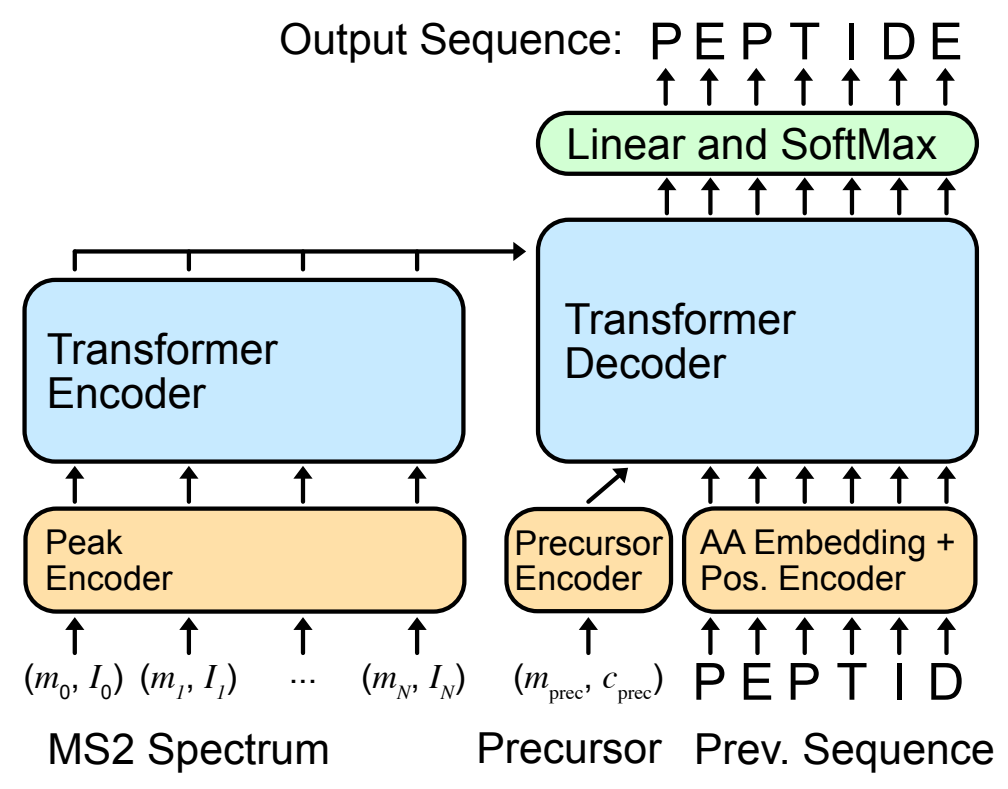

Figure 2: Casanovo model architecture with inputs and outputs

by projection to $d$ dimensions through a linear layer, after which the $m / z$ and intensity embeddings are summed to produce the input peak embedding. We also experiment in Section 5.3 with encoding intensity using a fixed, sinusoidal position embedding and concatenating it with the $m / z$ embedding.

Precursor information, used as input to the decoder, consists of the total mass $m_{\text {prec }} \in R$ and charge state $c_{\text {prec }} \in\{1, \ldots, 10\}$ associated with the spectrum. We use the same sinusoidal position embedding as peak $m / z$ 's for $m_{\text {prec }}$; $c_{\text {prec }}$ is embedded using an embedding layer, and the embeddings are summed to obtain the input precursor embedding. Preceding amino acids in the peptide sequence, another decoder input, are also encoded as the sum of an amino acid embedding and a sinusoidal position embedding of their position in the sequence.

\subsubsection{Training and inference strategy}

Taking the previously described embeddings as input, the transformer outputs scores which are treated as a probability distribution over the amino acid vocabulary for the next position in the sequence at each decoding step. The amino acid vocabulary includes 20 canonical amino acids, post-translationally modified versions of three of them (oxidation of methionine and deamidation of asparagine or glutamine), plus a special stop token to signal the end of decoding, yielding a total of 24 tokens. During training, the decoder is fed the amino acid prefix for the ground truth peptide following the teacher forcing paradigm 23. Cross-entropy between the model output probabilities and a binary matrix representing amino acid sequence of the ground truth peptide is minimized as the objective function. During inference, the highest scoring amino acid is predicted for each position in the sequence, and the decoder is fed its previous amino acid predictions at each decoding step. The decoding is finished either when the stop token is predicted or the pre-defined maximum peptide length of $\ell=100$ amino acids is reached.

\subsubsection{Model and training hyperparameters}

We train models with nine layers, embedding size $d=512$, and eight attention heads, yielding a total of $\sim 47 \mathrm{M}$ model parameters. A batch size of 32 spectra and $10^{-5}$ weight decay is used during training, with a peak learning rate of $5 \times 10^{-4}$. The learning rate is linearly increased from zero to its peak value in $100 \mathrm{k}$ warm-up steps, followed by a cosine shaped decay. Models are trained on 2 RTX 2080 GPUs for 30 epochs, which takes approximately two days, and model weights from the epoch with the lowest validation loss were selected for testing. These model hyperparameters - number of layers, embedding size, number of attention 
heads, and learning rate schedule - are used for all downstream experiments unless otherwise specified.

\subsubsection{Precursor $m / z$ filtering}

A critical constraint in de novo peptide sequencing requires the relative difference between total mass of the predicted peptide $m_{\text {pred }}$ and the observed precursor mass $m_{\text {prec }}$ of the spectrum to be less than a threshold value $\epsilon$ (specified in ppm) for the predicted sequence to be plausible: $\Delta m_{p p m}=\frac{\left|m_{\text {prec }}-m_{\text {pred }}\right| \times 10^{6}}{m_{\text {prec }}}<\epsilon$ In addition to providing precursor information as an input for the model to learn from, we filter out peptide predictions that do not satisfy the above constraint. The threshold value $\epsilon$ is a property of the mass spectrometer that the data is collected with, and hence is known at inference time. Accordingly, we choose $\epsilon$ based on the precursor mass error tolerance used in the database search to obtain ground truth peptide sequences for the test data.

Casanovo's source code and trained model weights are available as open-source under the Apache 2.0 license at https://github.com/Noble-Lab/casanovo

\subsection{Data set}

To evaluate the performance of Casanovo and compare it with state-of-the-art de novo peptide sequencing methods, we use the nine-species benchmark data set and evaluation framework first introduced by [2] and used in several subsequent studies [10, 12. This data set combines a total of about 1.5 million mass spectra from nine different experiments, each using the same instrument to analyze peptides from a different species. Based on database search identification, each spectrum comes with an assigned peptide sequence which is treated as ground truth to train and evaluate the methods. With approximately 300,000 unique peptide sequences in the data set, each sequence has around five spectrum instances on average, but around $40 \%$ of all peptide sequences have a single spectrum associated with them. Following [2], we employ a leave-one-out cross validation framework where we train a model on eight species and test on the held-out species for each of the nine species in the data set. In each case, we split the training set 90/10 for training and validation. This cross-species evaluation framework allows for testing the model on out-of-distribution samples, because the peptides in the training set are almost completely disjoint from the peptides of the held-out species. Out-of-distribution testing is particularly important for de novo sequencing models because most practical applications of de novo sequencing require models to perform well on spectra with never-before-seen peptide sequences.

\subsection{Evaluation metrics}

We use established performance measures - precision and recall calculated at the amino acid and peptide levels [2, 6, 8, - to evaluate the quality of a given model's predictions. In each case, for each spectrum we compare the predicted sequence to the ground truth peptide from the database search. Following [2], for the amino acid-level measures we first calculate the number $N_{\text {match }}^{a}$ of matched amino acid predictions, defined as all predicted amino acids which (1) differ by $<0.1 \mathrm{Da}$ in mass from the corresponding ground truth amino acid, and (2) have either a prefix or suffix that differs by no more than $0.5 \mathrm{Da}$ in mass from the corresponding amino acid sequence in the ground truth peptide. We then define amino acid-level precision as $N_{\text {match }}^{a} / N_{\text {pred }}^{a}$, where $N_{\text {pred }}^{a}$ is the number of predicted amino acids, and we define recall as $N_{\text {match }}^{a} / N_{\text {orig }}^{a}$, where $N_{\text {orig }}^{a}$ is the number of amino acids in the gold standard. For peptide predictions, a predicted peptide is considered a correct match if all of its amino acids are matched. Among a collection of $N_{\text {orig }}^{p}$ spectra, if our model makes predictions on a subset of $N_{\text {pred }}^{p}$ and correctly predicts $N_{\text {match }}^{p}$ peptides, we define peptide-level precision as $N_{\text {match }}^{p} / N_{\text {pred }}^{p}$ and recall as $N_{\text {match }}^{p} / N_{\text {orig }}^{p}$. To plot a precision-recall curve, we sort predictions by the confidence score provided by the model. Casanovo directly outputs amino acid-level confidence scores, and we use the mean score over all amino acids as a peptide-level confidence score. Once the model makes predictions for all spectra, $N_{\text {pred }}^{p}$ is equal to $N_{\text {orig }}^{p}$ and the precision-recall curve always terminates on the line $y=x$. This is not the case at the amino acid level because some predicted peptides differ in length from the corresponding gold standard peptides. 
Table 2: Empirical comparison of Casanovo, DeepNovo and PointNovo. The table lists the peptidelevel and amino acid-level precision and recall of three competing models on all nine benchmark cross-validation folds. Each fold's test set contains spectra from a single species, with a nearly disjoint sets of peptides between species.

\begin{tabular}{l|cccc|cccccc}
\hline & \multicolumn{4}{|c|}{ Peptide-level performance } & \multicolumn{4}{c}{ Amino acid-level performance } \\
Species & DeepNovo & PointNovo & Casanovo & \multicolumn{3}{c}{ DeepNovo } & PointNovo & \multicolumn{2}{c}{ Casanovo } \\
Prec=Recall & Prec=Recall & Prec & Recall & Prec & Recall & Prec & Recall & Prec & Recall \\
\hline Mouse & 0.286 & 0.355 & 0.661 & 0.350 & 0.623 & 0.627 & 0.626 & 0.633 & 0.890 & 0.430 \\
Human & 0.293 & 0.351 & 0.673 & 0.311 & 0.610 & 0.616 & 0.606 & 0.617 & 0.892 & 0.348 \\
Yeast & 0.462 & 0.534 & 0.851 & 0.561 & 0.750 & 0.750 & 0.779 & 0.781 & 0.965 & 0.576 \\
$M$. mazei & 0.422 & 0.478 & 0.767 & 0.488 & 0.694 & 0.692 & 0.712 & 0.716 & 0.932 & 0.520 \\
Honeybee & 0.330 & 0.396 & 0.733 & 0.360 & 0.630 & 0.635 & 0.644 & 0.652 & 0.916 & 0.395 \\
Tomato & 0.454 & 0.513 & 0.773 & 0.464 & 0.731 & 0.730 & 0.733 & 0.738 & 0.934 & 0.476 \\
Rice bean & 0.436 & 0.511 & 0.808 & 0.423 & 0.679 & 0.681 & 0.730 & 0.732 & 0.927 & 0.421 \\
Bacillus & 0.449 & 0.518 & 0.803 & 0.456 & 0.742 & 0.741 & 0.768 & 0.768 & 0.938 & 0.460 \\
Clam bacteria & 0.253 & 0.298 & 0.710 & 0.352 & 0.602 & 0.600 & 0.589 & 0.589 & 0.913 & 0.368 \\
\hline
\end{tabular}

\subsection{Chimericity score}

When we treat the peptide sequences identified by database search as the ground truth, we assume that each spectrum is generated by a single peptide. However, the mass spectrometer sometimes isolates a heterogeneous population of peptides for fragmentation. The result is a chimeric spectrum that contains peaks corresponding to fragment ions from multiple generating peptides. As a result, a predicted sequence can disagree with the ground truth sequence for two different reasons: either the prediction is incorrect, or the prediction corresponds to a second generating peptide that was isolated and fragmented alongside the gold standard peptide.

We therefore propose a chimericity score that aims to evaluate the likelihood that an apparent misprediction may instead represent detection of a chimeric spectrum. The intuition behind the score is that a truly chimeric spectrum will match b- and y-ions of both the gold standard and the predicted peptides. Given a spectrum $S$ and two peptides, $P_{k}$ and $P_{\ell}$, the chimericity score is

$$
\chi\left(S, P_{k}, P_{\ell}\right)=2 \min \left(\frac{n_{k}}{n_{k}+n_{\ell}+n_{c}}, \frac{n_{\ell}}{n_{k}+n_{\ell}+n_{c}}\right)
$$

where $n_{k}$ and $n_{\ell}$ denote the number of observed peaks uniquely matched to $P_{k}$ or $P_{\ell}$, respectively, and $n_{c}$ signifies the number of common peaks matching both peptides. This score ranges from zero to one, where zero denotes no evidence of chimericity - all peaks are explained by just one of the peptides - and a score of one strongly suggests a chimera because the matched peaks are split equally between the two peptide sequences.

\section{Results}

\subsection{Casanovo outperforms state-of-the-art methods}

We begin by using the previously described experimental setup and evaluation metrics (Sections 4.24 .3 to evaluate Casanovo's performance relative to two state-of-the-art neural network-based methods, DeepNovo 2 and PointNovo [10. In this comparison, peptide-level accuracy metrics are the primary quantifier of the sequencing model's practical utility, since the goal is to assign a complete peptide sequence to each observed spectrum. To characterize the performance of DeepNovo and PointNovo, we rely on the pre-trained weights of the former and the published results of the latter [10, since neither PointNovo's pre-trained weights nor its predictions for the benchmark data set are available.

At the peptide level, Casanovo substantially outperforms both previous methods across all species, with a mean improvement of 0.377 and 0.314 in precision relative to DeepNovo and PointNovo, respectively, at roughly comparable recall (Table 22. Note that the precision and recall values are identical to one another for both DeepNovo and PointNovo because $N_{\text {pred }}^{p}=N_{\text {orig }}^{p}$, whereas the precursor $\mathrm{m} / z$ filtering leads to $N_{\text {pred }}^{p}<N_{\text {orig }}^{p}$ for Casanovo. The peptide-level precision-recall curves (Figure 3 ) show that Casanovo 

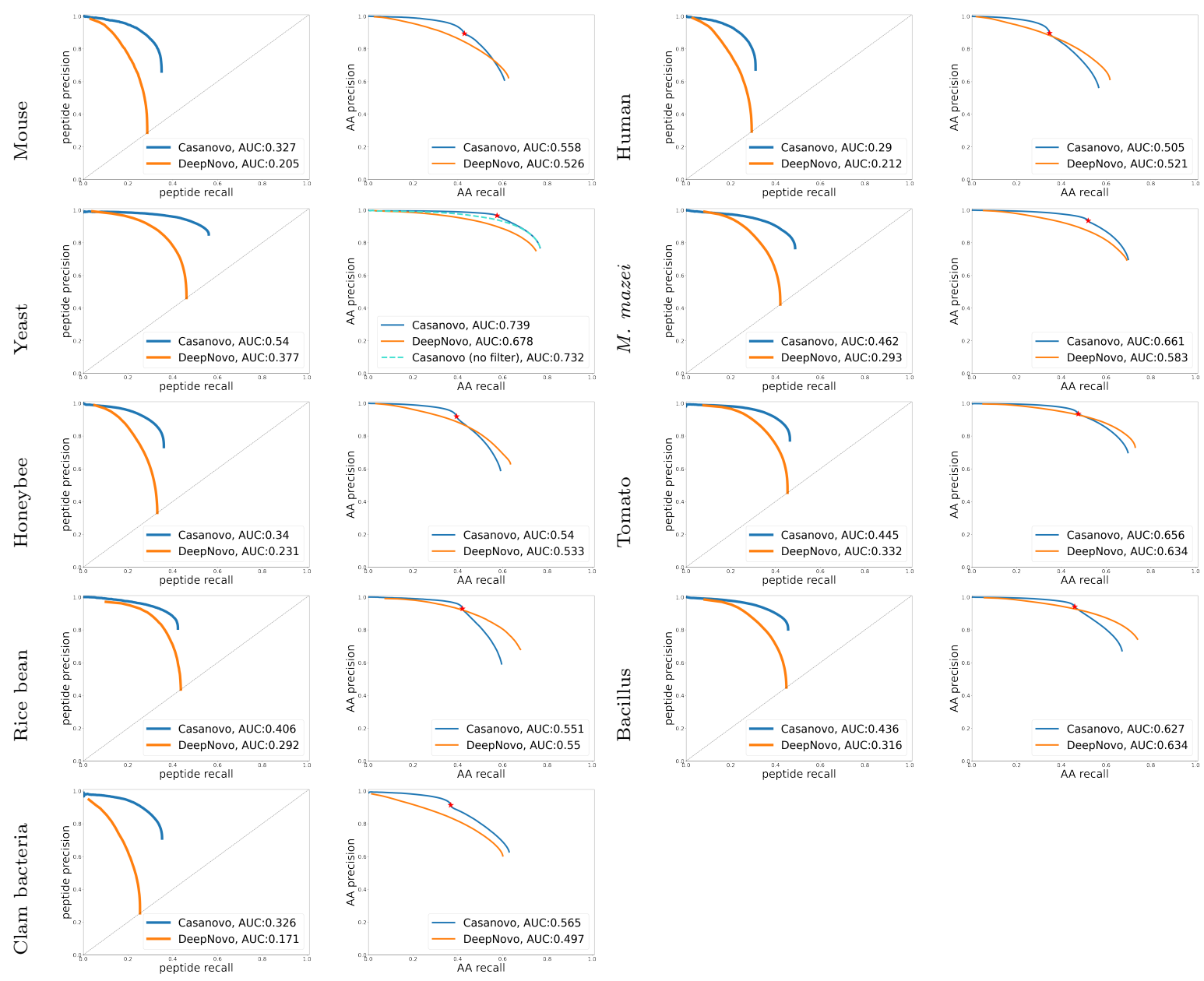

Figure 3: Precision-recall curves for Casanovo and DeepNovo. Curves are shown for each species (row) at the peptide level (left column) and amino acid level (right column). Curves are computed by sorting predicted peptides according to their confidence scores. For the amino acid level curves, all amino acids within a given peptide receive equal scores. The peptide-level curves of DeepNovo do not extend to the top left corner because many predictions are assigned maximum confidence. For Casanovo at the amino acid level, all amino acids from peptides that pass the precursor $\mathrm{m} / z$ filtering are ranked above those from peptides that do not pass the filter, and the boundary between the two is indicated by a point on each curve. For yeast, we also include a second amino acid-level curve for Casanovo, in which the precursor $m / z$ filtering is omitted. 
A

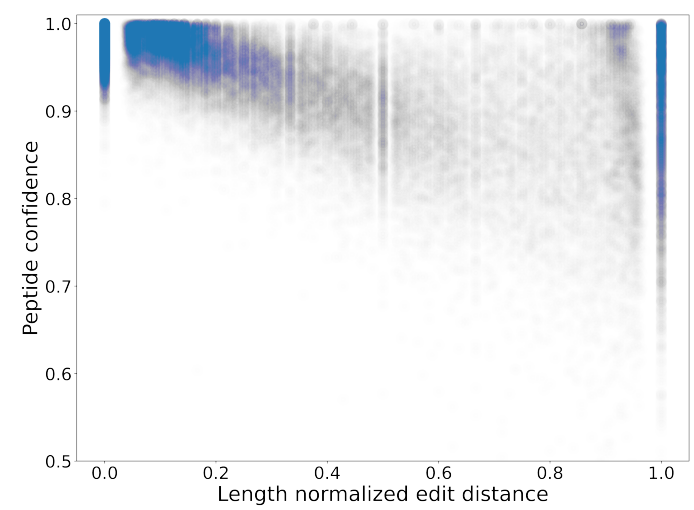

$\mathrm{B}$

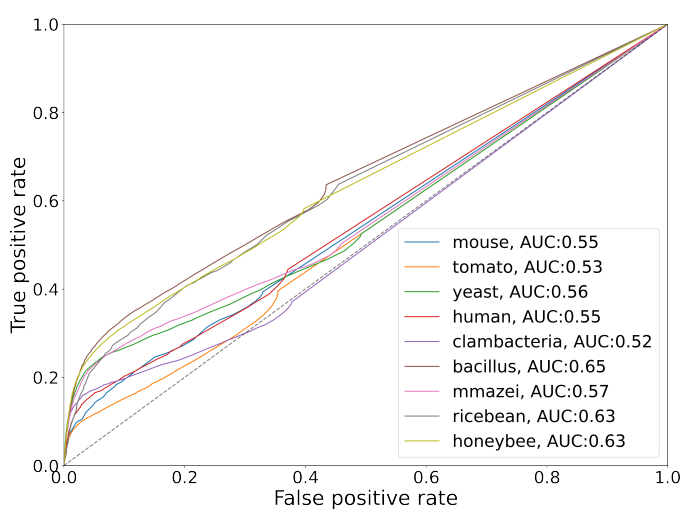

Figure 4: Chimericity analysis (A) Casanovo peptide confidence as a function of length-normalized edit distance. (B) ROC curves, one per species, illustrating the difference in chimericity score distributions between Group 1 (spectra mis-predicted by Casanovo but not DeepNovo) and Group 2 (spectra mis-predicted by DeepNovo but not Casanovo). Each curve ends with a straight line corresponding to all spectra with chimericity scores of zero.

consistently outperforms DeepNovo over a range of peptide confidence thresholds. This trend is also reflected by the area under the PR curve (AUPRC) metric (Figure 3), with Casanovo outperforming DeepNovo by 0.124 on average.

Similarly, at the amino acid level, Casanovo outperforms DeepNovo and PointNovo, particularly in the high-precision portions of the curves. As expected, the precursor $m / z$ filtering, which prioritizes predicting full peptide sequences with high precision over partially correct peptide predictions, yields better precision at the cost of reduced recall. In all nine species, the point on the Casanovo curve corresponding to the filter lies above the DeepNovo precision-recall curve, and in seven of the nine species Casanovo's AUPRC exceeds DeepNovo's. We further discuss the effects of precursor $\mathrm{m} / z$ filtering in Section 5.3 .

Complementing its improved de novo peptide sequencing performance, Casanovo achieves these results with fewer model parameters $(47 \mathrm{M})$ than DeepNovo $(86 \mathrm{M})$. (The number of parameters and model dimensions were not reported for PointNovo.) Casanovo also runs inference at a faster rate of 119 spectra/s on an RTX 2080 compared to DeepNovo's 36 spectra/s and PointNovo's reported 20 spectra/s on an RTX 2080 Ti (a comparatively faster GPU) [10].

\subsection{Casanovo's mispredictions are enriched for potential chimeras}

To better understand Casanovo's behavior, we calculate the peptide length-normalized count of non-matching amino acids in each predicted peptide, i.e. the normalized edit distance, and plot it against the peptide confidence reported by the model (Figure 44). Not surprisingly, we see relatively high confidence scores associated with correct predictions (edit distance of zero) and a trend of decreasing peptide confidence as a function of edit distance. However, we also observe a cluster of high confidence predictions for many peptides with high edit distance (top right corner of the plot). This observation led us to investigate whether a subset of the nominally incorrect Casanovo predictions may be associated with chimeric spectra. In particular, we pursued the hypothesis that, relative to DeepNovo (the only other method for which we have detailed predictions), Casanovo's nominally incorrect predictions are more highly enriched for potential chimeras.

Accordingly, we score the incorrect predictions made by both DeepNovo and Casanovo using the chimericity score (Equation 21. In particular, we focus on two groups of interest: Group 1 contains spectra with nominally correct DeepNovo predictions and incorrect Casanovo predictions, and Group 2 is the opposite. To quantify the difference between these two chimericity score distributions, we rank spectra based on their chimericity scores and plot an ROC curve, one per species (Figure 4B). The curves show that higher chimericity scores consistently distinguish Group 1 from Group 2, with an AUROC greater than 0.5 across species. We also observe that the mean chimericity of Group 1 is twice as large as Group 2's (0.217 versus 0.109), with an even higher mean score of 0.742 among the subsample of spectra with normalized edit distance of 1 . These 


\begin{tabular}{lcccc}
\hline \multirow{2}{*}{ Model variant } & \multicolumn{2}{c}{ Peptide } & \multicolumn{2}{c}{ Amino acid } \\
& Precision & Recall & Precision & Recall \\
\hline Standard Casanovo & 0.851 & 0.561 & 0.965 & 0.576 \\
No precursor $m / z$ filtering & 0.561 & 0.561 & 0.769 & 0.768 \\
DP post-processing & 0.565 & 0.565 & 0.726 & 0.723 \\
Focal loss & 0.802 & 0.532 & 0.938 & 0.543 \\
$I \times m / z$ embedding & 0.736 & 0.446 & 0.841 & 0.463 \\
Sinusoidal $I$ embedding & 0.817 & 0.538 & 0.943 & 0.552 \\
\hline
\end{tabular}

Table 3: Performance comparison of different Casanovo variants. All results are for the yeast test set.

results suggest that the reported evaluation metrics, which assume that each spectrum has a single generating peptide, likely underestimate Casanovo's performance advantage relative to DeepNovo.

\subsection{Ablation and variation experiments}

One of the key components of DeepNovo, and its successor PointNovo, is a post-processor that uses the knapsack dynamic programming algorithm to ensure that the mass of the predicted peptide is close to the observed precursor mass. Ablation experiments in the DeepNovo experiment paper show that removing this component leads to a decrease in peptide-level precision (and, by definition, recall) of $12.4 \%$ (averaged over test sets) 22. Accordingly, we tested three variants of Casanovo on the yeast species test set: no post-processor, the knapsack post-processor, and our simple $m / z$ filter (Table 3).

At the peptide level, we observe a much smaller benefit from the knapsack algorithm - an increase in the peptide-level precision and recall from 0.561 to 0.565 - than was reported in the DeepNovo paper. On the other hand, the simple $m / z$ filter keeps the recall at 0.561 but yields a very large increase in precision, from 0.561 to 0.851 .

At the amino acid level, the effects of these two post-processors is different. Relative to Casanovo with no post-processing, applying the knapsack algorithm yields small decreases in both precision $(0.769 \rightarrow 0.726)$ and recall $(0.768 \rightarrow 0.723)$, whereas adding the precursor $\mathrm{m} / z$ filtering yields much higher precision $(0.769$ $\rightarrow 0.965)$ and much lower recall $(0.768 \rightarrow 0.576)$. A qualitative review of peptide predictions suggests that incorrect amino acid predictions in earlier decoding steps cause the post-processor to discard correct amino acids from among options in later decoding steps, leading to a drop in amino acid-level performance. This is supported by the plot of the PR curve with and without the precursor $m / z$ filter (Figure 3, yeast data), where we see that the effect of the filter is to boost precision along the entire curve.

Finally, we train three additional variants of Casanovo, none of which provides any performance improvements over the standard model (Table 3). The first variant uses a focal loss function, adopted from [10], instead of cross entropy. We also investigated two alternate methods of peak embedding. The first, also adopted from PointNovo, replaces summation of $\mathrm{m} / z$ and $I$ embeddings with direct multiplication of the $I$ value and the $m / z$ embedding. The second peak embedding strategy implements a sinusoidal encoding for $I$, similar to the $m / z$ embedding, although using only 32 dimensions, and concatenates $I$ with the $m / z$ embeddings instead of summing the two.

\section{Discussion}

Prior work in de novo peptide sequencing has used deep learning models that combine separate neural network architectures followed by complex post-processing steps. Our approach, Casanovo, leverages the transformer architecture to produce a unified solution to translate mass spectra directly into peptide sequences, without resorting to discretization of the spectrum $\mathrm{m} / \mathrm{z}$ axis and without complex post-processing. We find that Casanovo achieves state-of-the-art performance on the standard benchmark data set, with fewer model parameters compared to existing methods.

Casanovo's inference speed is fast enough to allow real time de novo sequencing, i.e., sequencing at the speed that the mass spectrometer generates spectra, raising the possibility of helping guide mass spectrometry experiments as they are being conducted [9. In practice, real-time search results can be useful 
for making decisions about peptide elution order 24, improving the accuracy of stable isotope labeling [25], post-translational modification site localization [25], or deciding whether to trigger an MS3 (secondary fragmentation) scan [26].

Casanovo improves substantially over the previous state of the art in terms of peptide-level precision, but this leaves a significant portion of the test spectra without plausible predictions. Clearly, exploring methods to find good predictions for these spectra is an avenue for future research. To explore the potential benefit of such an approach, we combined Casanovo and DeepNovo predictions by inserting DeepNovo predictions whenever the $\mathrm{m} / z$ filter eliminates a Casanovo prediction. The resulting model achieves up to $10 \%$ higher peptide recall than Casanovo and exceeds the previous state-of-the-art method, PointNovo, on all four evaluation metrics across species. This observation suggests that precursor information should be included as a stronger prior in modeling mass spectra. A straightforward approach might involve choosing among a larger set of peptide candidates generated by beam search during inference, with a constraint on the predicted mass. Alternatively, Casanovo's loss function could be modified to penalize peptide predictions which do not match the precursor mass.

\section{References}

[1] M. M. VanDuijn, L. J. Dekker, W. F. van Ijcken, P. A. E. S. Smitt, and T. M. Luider. Immune repertoire after immunization as seen by next-generation sequencing and proteomics. Frontiers in Immunology, 8:1286, 2017.

[2] N. H. Tran, X. Zhang, L. Xin, B. Shan, and M. Li. De novo peptide sequencing by deep learning. Proceedings of the National Academy of Sciences of the United States of America, 31:8247-8252, 2017.

[3] R. L. Mayer and F. Impens. Immunopeptidomics for next-generation bacterial vaccine development. Trends in Microbiology, 29(11):1034-1045, 2021.

[4] T. Muth, D. Benndorf, U. Reichl, E. Rapp, and L. Martens. Searching for a needle in a stack of needles: challenges in metaproteomics data analysis. Molecular Biosystems, 9(4):578-585, 2013.

[5] J. A. Taylor and R. S. Johnson. Sequence database searches via de novo peptide sequencing by tandem mass spectrometry. Rapid Communications in Mass Spectrometry, 11:1067-1075, 1997.

[6] B. Ma, K. Zhang, C. Hendrie, C. Liang, M. Li, A. Doherty-Kirby, and G. Lajoie. PEAKS: powerful software for peptide de novo sequencing by tandem mass spectrometry. Rapid Communications in Mass Spectrometry, 17(13):2337-2342, 2003.

[7] V. Dancik, T.A. Addona, K.R. Clauser, J.E. Vath, and P.A. Pevzner. De novo peptide sequencing via tandem mass spectrometry. Journal of Computational Biology, 6(3-4):327-342, 1999.

[8] A. Frank and P. Pevzner. Pepnovo: de novo peptide sequencing via probabilistic network modeling. Analytical Chemistry, 77:964-973, 2005.

[9] B. Ma. Novor: Real-time peptide de novo sequencing software. Journal of the American Society for Mass Spectrometry, 26:1885-1894, 2015.

[10] R. Qiao, N. H. Tran, L. Xin, X. Chen, M. Li, B. Shan, and A. Ghodsi. Computationally instrumentresolution-independent de novo peptide sequencing for high-resolution devices. Nature Machine Intelligence, 3:420-425, 2021.

[11] H. Yang, H. Chi, W. Zeng, W. Zhou, and S. He. pNovo 3: precise de novo peptide sequencing using a learning-to-rank framework. Bioinformatics, 35(14):i83-i90, 2019.

[12] K. Karunratanakul, H.-Y. Tang, D. W. Speicher, E. Chuangsuwanich, and S. Sriswasdi. Uncovering thousands of new peptides with sequence-mask-search hybrid de novo peptide sequencing framework. Molecular and Cellular Proteomics, 18:2478-2491, 2019. 
[13] W. Bittremieux. spectrum_utils: A python package for mass spectrometry data processing and visualization. Analytical Chemistry, 92(1):659-661, 2020.

[14] H. Steen and M. Mann. The ABC's (and XYZ's) of peptide sequencing. Nature Reviews Molecular Cell Biology, 5:699-711, 2004.

[15] W. S. Noble and M. J. MacCoss. Computational and statistical analysis of protein mass spectrometry data. PLOS Computational Biology, 8(1):e1002296, 2012.

[16] A. I. Nesvizhskii. A survey of computational methods and error rate estimation procedures for peptide and protein identification in shotgun proteomics. Journal of Proteomics, 73(11):2092 - 2123, 2010.

[17] Bernd Fischer, Volker Roth, Joachim M Buhmann, Jonas Grossmann, Sacha Baginsky, Wilhelm Gruissem, Franz Roos, and Peter Widmayer. A hidden Markov model for de novo peptide sequencing. Advances in Neural Information Processing Systems, 17:457-464, 2005.

[18] X. Zhou, W. Zeng, H. Chi, C. Luo, C. Liu, J. Zhan, S. M. He, and Z. Zhang. pDeep: predicting MS/MS spectra of peptides with deep learning. Analytical Chemistry, 89(23):12690-12697, 2017.

[19] C. R. Qi, H. Su, K. Mo, and L. J. Guibas. PointNet: deep learning on point sets for 3D classification and segmentation. In Proceedings of the IEEE Conference On Computer Vision and Pattern Recognition, pages 652-660, 2016.

[20] A. Vaswani, N. Shazeer, N. Parmar, J. Uszkoreit, L. Jones, A. N. Gomez, Ł. Kaiser, and I. Polosukhin. Attention is all you need. Advances in Neural Information Processing Systems, 30, 2017.

[21] A. Rives, J. Meier, T. Sercu, S. Goyal, Z. Lin, J. Liu, D. Guo, M. Ott, C. L. Zitnick, J. Ma, and R Fergus. Biological structure and function emerge from scaling unsupervised learning to 250 million protein sequences. Proceedings of the National Academy of Sciences of the United States of America, 118(15):e2016239118, 2021.

[22] Z. Avsec, V. Agarwal, D. Visentin, J. R. Ledsam, A. Grabska-Barwinska, K. R. Taylor, Y. Assael, J. Jumper, P. Kohli, and D. R. Kelley. Effective gene expression prediction from sequence by integrating long-range interactions. Nature Methods, 18:1196-1203, 2021.

[23] R. J. Williams and D. Zipser. A learning algorithm for continually running fully recurrent neural networks. Neural Computation, 1(2):270-280, 1989.

[24] D. K. Bailey, M. T. McDevitt, M. C. Westphall, D. J. Pagliarini, and J. J. Coon. Intelligent data acquisition blends targeted and discovery methods. Journal of Proteome Research, 13:2152-2161, 2014.

[25] D. K. Bailey, C. M. Rose, G. C. McAlister, J. Brumbaugh, P. Yu, C. D. Wenger, M. C. Westphall, J. A. Thomson, and J. J. Coon. Instant spectral assignment for advanced decision tree-driven mass spectrometry. Proceedings of the National Academy of Sciences of the United States of America, 109(22):8411-8416, 2012.

[26] D. K. Schweppe, J. K. Eng, D. Bailey, R. Rad, Q. Yu, J. Navarrete-Perea, E. L. Huttlin, B. K. Erickson, J. A. Paolo, and S. P. Gygi. Full-featured, real-time database searching platform enables fast and accurate multiplexed quantitative proteomics. Journal of Proteome Research, 19(5):2026-2034, 2020. 